\title{
Dissipation effects in superconducting heterostructures with tungsten nanorods as weak links
}

\author{
V.E. Shaternik ${ }^{1}$, A.P. Shapovalov ${ }^{2}$, O.Yu. Suvorov ${ }^{1}$, E.S. Zhitlukhina ${ }^{3,4}$, \\ M.A. Belogolovskii ${ }^{1,4}$, P. Febvre ${ }^{5}$, and A.A. Kordyuk ${ }^{1}$ \\ ${ }^{1}$ G.V. Kurdyumov Institute for Metal Physics, National Academy of Sciences of Ukraine \\ 36 Academician Vernadsky Blvd., Kyiv 03142, Ukraine \\ E-mail: shat@imp.kiev.ua \\ ${ }^{2}$ V.N. Bakul Institute for Superhard Materials, National Academy of Sciences of Ukraine \\ 2 Avtozavodskaya Str., Kyiv 04074, Ukraine \\ ${ }^{3}$ O.O. Galkin Donetsk Institute for Physics and Engineering, National Academy of Sciences of Ukraine \\ 46 Nauki Ave., Kyiv 03028, Ukraine \\ ${ }^{4}$ Vasyl' Stus Donetsk National University, 21 600-richya Str., Vinnytsia 21021, Ukraine \\ ${ }^{5}$ Superconducting Electronics Group, IMEP-LAHC - CNRS UMR 5130, Université Savoie Mont Blanc \\ Le Bourget du Lac Cedex 73376, France
}

Received November 24, 2017, published online January 25, 2018

\begin{abstract}
Thin-film hybrid heterostructures formed by superconducting molybdenum-rhenium-alloy films with a critical temperature of about $9 \mathrm{~K}$ and nanoscale silicon-based semiconducting interlayers with metallic tungsten nanorods have been fabricated and studied. Current-voltage characteristics of the junctions were measured at $4.2 \mathrm{~K}$ and under influence of $11 \mathrm{GHz}$ microwave irradiation. The evidence of a quasi-one-dimensional transport through the tungsten weak links disrupted by phase-slip centers was revealed in MoRe/doped Si/MoRe trilayers under irradiation by a high-frequency field. Also, measured current-voltage characteristics of five-layer MoRe/doped Si/MoRe/doped Si/MoRe devices exhibit a strong influence of a dissipation state in the MoRe interlayer. Namely, the switching from a superconducting state with low dissipation to a finite-conductance regime can be initiated by the emergence of an extra phase-slip center in the MoRe interlayer. Possible physical mechanisms of the two findings are discussed.
\end{abstract}

PACS: 85.25.Cp Josephson devices;

73.21.Hb Quantum wires;

74.40.Gh Nonequilibrium superconductivity.

Keywords: Josephson junction, weak link, quasi-one-dimensional transport, phase-slip events, microwave irradiation.

\section{Introduction}

Bottom-up fabrication of electronic devices from predefined nanoscale structures is one of the major activities in nanoelectronics. In particular, it relates hybrid superconductor-semiconductor samples which are considered to be potential building blocks for novel low-temperature devices [1]. Up to date, there remains a lot of challenges to scale down the related heterostructures and find a way to integrate them with mainstream silicon technology. In our previous works [2-4], we have proposed a novel promising approach for creating Josephson weak-link junctions based on self-assembled tungsten drops within a nanoscale silicon interlayer between two superconducting MoRe-alloy films that has been inspired by earlier publications about $\mathrm{Nb}$ /doped amorphous Si/Nb trilayers [5,6]. We have shown the ability to carry charge currents with zero dissipation through the MoRe/Si(W)/MoRe junctions and revealed nonhysteretic current-voltage $(I-V)$ characteristics with steplike response to the microwave irradiation [2]. In the present paper, we discuss a physical mechanism leading to the dissipative (resistive) state arising in our MoRe/Si(W)/MRe 
samples above a threshold supercurrent value $I_{C}$, which is below the depairing current. Since the pair current of measured samples is carried out by ultra-narrow metallic channels $[4,7]$ we propose a phase-slip paradigm to explain specific features of the observed $I-V$ curves.

Phase slips (PS) are time-dependent elementary excitations of the superconducting order parameter corresponding to a local suppression of its amplitude and a simultaneous jump of the phase by $\delta \varphi=2 \pi$ [8]. Historically they were predicted as thermally activated topological defects in quasi-one-dimensional superconducting samples at temperatures slightly lower than the critical one $T_{C}$ [9]. Later it was recognized that PS centers may occur also far below $T_{c}$, due to quantum tunneling events [10]. A superconducting wire with a phase slip $\delta \varphi$ (or a series of $n$ such structures) is in a metastable state with lower velocity. It reveals a finite voltage drop due to the Josephson relation $(2 e / \hbar) V=d(\delta \varphi) / d t$. At zero bias current, symmetric distribution of $2 \pi n$ and $-2 \pi n$ phase-slip events takes place but for a finite electric current $I$ one of the two events becomes more favored, leading to power dissipation. If heat removal is efficient, the temperature increase (even at the phase-slip central part) is not significant and the supercurrent through such a system is stabilized [11]. At first, a single PS is expected to arise and, in long nanowires where several PSs may coexist, further current increase causes new voltage steps in approaching the normal state. In general, the multiple stepped $I-V$ characteristic is a common feature in the electrical transport property of quasi-one-dimensional (1D) superconducting systems and is usually interpreted as a result of spatially localized PS centers due to local defects or imperfections in a nanowire, see Ref. 8.

Up to now, the main discussion concerning the PS phenomenon was concentrated on initially homogeneous superconducting quasi-1D samples but, as was stressed in Ref. 12, the situation differs for spatially inhomogeneous systems, such as superconducting devices with weak links (nanobridges or Josephson junctions). The authors [12] have studied a $1 \mathrm{D}$ superconductor with a normal or weakly superconducting spatial inhomogeneity of the order of the coherence length $\xi(T)$ which was modeled by a coordinate- dependent critical temperature. They have found a currentinduced transition above a critical current $I_{C}$ from a stationary superconducting state to an inhomogeneous PS structure occurring instantly inside the weak link and we can expect that it may provide, at least, qualitative explanation of our experimental data for a weak link between the superconducting layers formed by $\mathrm{W}$ nanorods.

\section{Experiments}

In our experiments, we have used 100-nm thick MoRe films with $45 \% \mathrm{Re}$ as superconducting terminals. Due to the comparatively high critical temperature $T_{C}$ of about $9 \mathrm{~K}$ for conventional superconductors and good mechanical properties like hardness and mechanical strength, this material is very appropriate for hybrid superconducting heterostructures. The MoRe films were deposited onto dielectric substrates at ambient temperature using a dc magnetron sputtering technique in a vacuum chamber with argon at the pressure of $1 \mathrm{~Pa}$. To create an intermediate weak link between electrodes, we have used a target consisting of a pure Si wafer and a number of tungsten wires of $0.3 \mathrm{~mm}$ diameter. The tungsten concentration $n_{\mathrm{W}}$ in the $\mathrm{Si}(\mathrm{W})$ mixture was changed from 0 to 10 at.\%.

The sizes of the MoRe/doped Si/MoRe trilayers shown in Fig. 1(a) varied from $50 \times 50$ to $100 \times 100 \mu \mathrm{m}$. Critical current values $I_{C}$ were found to be up to several tens of $\mathrm{mA}$. Low-temperature transport measurements at $4.2 \mathrm{~K}$ were performed mainly with a voltage source, with the exception of microwave experiments that have been done with a current-source regime. In the latter case the samples were fixed into a resonator and irradiated by microwaves whose frequency $f$ was varied between 0.5 and $20 \mathrm{GHz}$, with microwave power varying from -40 to $20 \mathrm{~dB}$ around a nominal power. We have observed strong sensitivity of the critical current $I_{C}$ with the irradiation. The critical current decreased with increasing microwave power, up to its complete suppression.

When the tungsten content in $\mathrm{Si}(\mathrm{W})$ barriers reached 6-8 at.\%, the $I-V$ curves of the MoRe/Si(W)/MoRe samples revealed the presence of the superconducting current at (a)

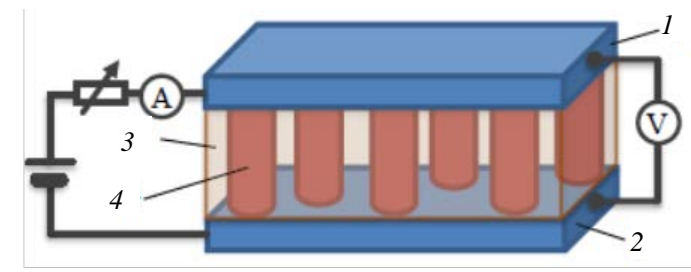

(b)

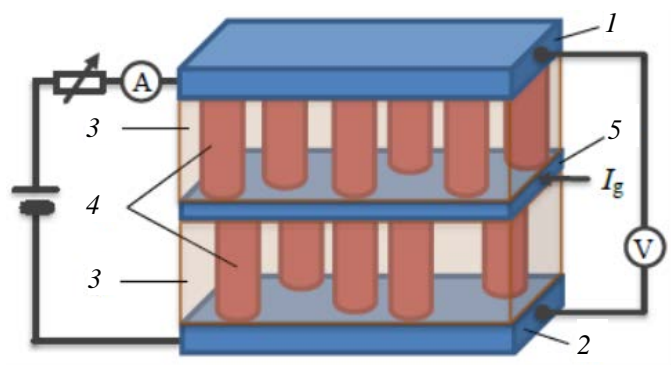

Fig. 1. (Color online) Schematic representation of a MoRe/Si(W)/MoRe trilayer (a) and a five-layer MoRe/Si(W)/MoRe/Si(W)/MoRe device (b) with two superconducting electrodes 1 and 2, a silicon-based barrier 3 with tungsten nanorods 4, and a MoRe interlayer 5 with the governing current $I_{g}$ through it. 
zero voltage bias and a large excess current, a shift of the linear region in the current-voltage characteristics with respect to those in the normal state, which is a signature of the Andreev-like reflection at the contacts between the nanorods and the superconducting electrodes $[3,4]$. In the following, we present our data obtained for $n_{\mathrm{W}}=10$ at.\%.

Besides conventional MoRe/Si(W)/MoRe trilayers (Fig. 1(a)) we have also fabricated more complicated fivelayer heterostructures with an auxiliary superconducting MoRe-alloy interlayer in the middle of the heterostructure, see Fig. 1(b). The main aim of the modification has been to create a controllable region inside the weak link with suppressed superconducting properties. To realize it, we have used a multi-terminal connection to an external circuit. Voltage and current leads applied to one set of four terminals controlled the superconducting state of the interlayer 5, see Fig. 1(b), whereas another set of terminals was used for measuring current-voltage characteristics of the entire fivelayer device (is shown in Figs. 1(a) and 1(b)).

As we have found in Ref. 4, during the deposition of the hybrid barrier, tungsten drops are self-organized into separate nanoclusters with dimensions on the order of $5 \cdot 10^{-2} \mu \mathrm{m}$ in diameter and an area up to $3 \cdot 10^{-3} \mu \mathrm{m}^{2}$ which are evenly distributed along the Si-based barrier. Some of them shown in Figs. 1(a) and 1(b) make direct contact between two neighboring superconductors and the transport through them determines the main characteristics of the devices.

In Fig. 2 we demonstrate a typical $I-V$ trace for a MoRe superconducting interlayer which exhibits a wide transition region from a persistent current to an ohmic behavior with two pronounced steps. As the bias current increased from zero, the sample switched to a first resistive state at the onset current $I_{C}$ corresponding to the emergence of a finite voltage during the upsweep ( $I_{C}$ was always less than the

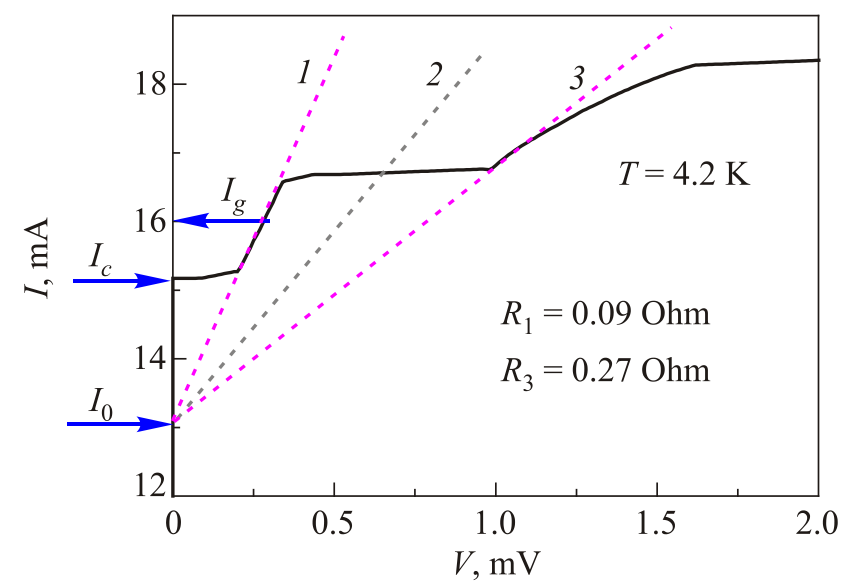

Fig. 2. (Color online) Typical current-vs-voltage dependence for a superconducting MoRe-alloy interlayer embedded between two semiconducting barriers in a five-layer MoRe/doped Si/MoRe/doped Si/MoRe heterostructure, $I_{g}$ is the governing current value for experiments with five-layer heterostructures, $T=4.2 \mathrm{~K}$. depairing critical current in the equilibrium state). At higher biases, the second voltage jump took place following a strongly nonlinear $I-V$ dependence. Due to the conventional explanation, such behavior in quasi-two-dimensional superconducting samples is generated by phase-slip fluctuations disrupting the persistent current and imposing a nonzero resistance to the studied films. It should be noticed that for superconducting samples with lateral dimensions larger than $\xi(T)$, as it is in our case, PS centers can be generalized to PS lines which have been discovered in the paper by I.M. Dmitrenko et al. [13]. It is clear now that oscillations of the order parameter in such topological fluctuations occur in the form of propagating waves carrying a zero-order parameter across the sample. Such waves known as kinematic vortices were observed experimentally in Ref. 14 and were revealed in numerical simulations [15].

Another mechanism for the resistive state of currentcarrying superconductors is the formation of hot spots, localized in normal regions where the local temperature exceeds the critical value, suppressing the superconducting condensate completely. As was found experimentally and confirmed by numerical calculations performed within the time-dependent Ginzburg-Landau theory, the PS regime in a superconducting strip is dynamically stable in time, whereas the hot-spot state, which always appears after the PS excitations have been nucleated, is characterized by the spread of the normal-state region until the whole sample switches to the normal state, see the related discussion and references in Ref. 15. Thus, we expect that the first resistive state seen in Fig. 2 with $R_{1}=0.09$ Ohm corresponds to a single phase-slip event. According to Ref. 14 characteristic features of the PS lines are (i) a voltage jump from zero voltage at some critical current; (ii) intersection of all resistive-branch slopes at one point on the current axis $I_{0}$ that can be interpreted as a time-averaged supercurrent $\left\langle I_{\mathrm{S}}(t)\right\rangle$; (iii) proportionality of the differential resistance $R_{n}$ of a resistive branch to its number $n: R_{n}=n R_{1}$. These conditions are fulfilled for two branches seen in Fig. 2. The lack of the second resistive branch can arise due to simultaneous generation of two PS lines. In our further experiments, we have fixed the governing current $I_{g}$ through the MoRe interlayer to $16 \mathrm{~mA}$ that corresponds to the first $(n=1)$ resistive state.

\section{Phase-slip events in multilayered superconducting structures: non-trivial results}

In the following, we limit ourselves to two unexpected results obtained for three- and five-layer heterostructures shown in Figs. 1(a) and 1(b), respectively. The first one was obtained by studying the effect of a high-frequency radiation applied to MoRe/doped Si/MoRe trilayers. An inductive loop was used to couple the radiation to the sample. Without the irradiation no stepped structure was revealed in the $I-V$ curves but, after the samples had been subjected to a microwave field, the voltage steps appeared. As can be 
seen from Fig. 3, an increase in the radiation-field power suppresses the critical dc current $I_{C}$ for a given bath temperature $4.2 \mathrm{~K}$. There are several mechanisms able to reduce the $I_{C}$ value, Joule heating, pair-breaking effects and so on. For $\mathrm{Pb}$ whiskers, it was found that the main factor is pair-breaking by the magnetic field of the high-frequency radiation [16].

The fact that a pure ac current can drive a quasi-1D superconductor into a dissipative phase-slip state was discussed in Ref. 11 within the framework of a time-dependent Ginzburg-Landau theory. From these calculations it follows that in the presence of dc and ac currents there is a critical value $I_{C}$ of the dc current up to which the homogeneous superconducting state exists. It is smaller than the Ginzburg-Landau critical current and depends on the amplitude of the ac current as well as on its frequency $f$ and material properties. By increasing the dc current, the superconducting nanowire enters the phase-slip state at the current value $I=I_{C}$ by a voltage jump to the first resistive
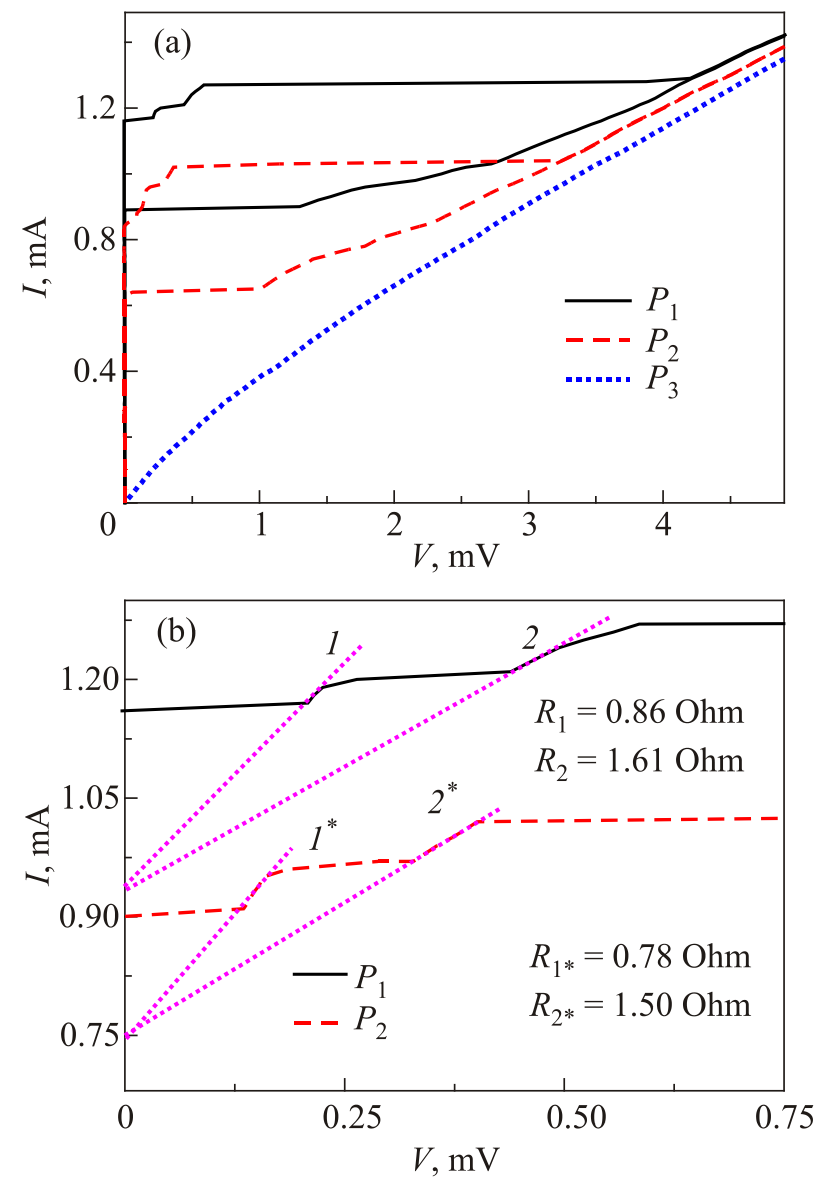

Fig. 3. (Color online) Effect of the high-frequency ( $f=11 \mathrm{GHz}$ ) irradiation on current-voltage curves of a MoRe/Si(W)/MoRe trilayer at various power levels $P_{1}<P_{2}<P_{3}$ (solid, dashed, and dotted lines, respectively). The $I-V$ curves demonstrate hysteretic behavior (a) and exhibit the emergence of phase-slip centres (b) for the first two $I-V$ curves with moderate microwave radiation, $T=4.2 \mathrm{~K}$. state with the resistance $R_{1}$ and after decreasing current the sample returns to a homogenous superconducting regime at the retrapping current value $I_{r}<I_{c}$, i.e., the transition from the homogeneous superconducting state into the dissipative phase-slip state is hysteretic, as can be seen from Fig. 3(a).

The authors [17] proposed the following analytic approximation for the time-dependent phase-slip center in a weak link:

$$
V(t)=R_{1}\left(I-I_{S}(t)\right),
$$

where the spatially averaged time-dependent supercurrent above $I_{C}$ is equal to $I_{S}(t)=a I_{C}(1+\cos \varphi(t))$ and $\varphi(t)$ is the quantum phase difference across the phase slip which satisfies the equation $d \varphi(t) / d t=2 e V(t) / \hbar$, the coefficient $a$ equals to 0.5 in the dirty case and increases to a value slightly below unity for a clean sample [11]. Thus,

$$
\varphi(t)=\varphi(0)+\frac{2 e}{\hbar} \int_{0}^{t} V\left(t^{\prime}\right) d t^{\prime} .
$$

If the time average of the voltage is nonzero, the phase difference and, as a consequence, a superfluid velocity proportional to the phase gradient increases as a function of time. To avoid it, in the stationary state the phase difference generated by the voltage should be destroyed by a phase-slip process when the critical current of a weakest point in the superconducting wire is reached. The quasiparticle excitations generated during the PS cycle in the core region diffuse into the surrounding superconductor. Quasielectron and quasi-hole ones are migrating in opposite directions creating the charge imbalance. The differential resistance developed by a PS center is given by

$$
R_{1}=2 \rho_{N} l_{Q} / A
$$

where $\rho_{N}$ is the normal-state resistivity, $l_{Q}=\sqrt{D \tau_{Q}}, D$ is the diffusion constant, $\tau_{Q}$ is the relaxation time for a process of reducing the difference between electrochemical potentials of pairs and quasi-particles, $A$ is the PS-center cross-sectional area. Taking into account that the resistivity of ultra-narrow $\mathrm{W}$ lines strongly increases due to the size effect [18], that $l_{Q}$ should be of the order of the distance between the MoRe layers and the total $\mathrm{W}$-wires area $A$ can be up to $1 \mu \mathrm{m}^{2}$, we obtain $R_{1} \approx 1$ Ohm that agrees well with the data shown in Fig. 3(b). As follows from Fig. 3(b), the two resistive branches meet the main criteria [14] for the PS events, namely, $R_{2} \approx 2 R_{1}$ and the intersection of corresponding straight lines occurs at the current axis. Under the microwave irradiation with $P_{1}$ and $P_{2}$ powers, the ratio of two currents $I_{C} / I_{0} \approx 0.8$ is almost the same since it is mainly determined by the purity of the $S$ nanowire as was shown for a homogeneous one-dimensional superconductor in Ref. 11. The first phase-slip resistance value $R_{1}$ which depends only on the material properties of the su- 


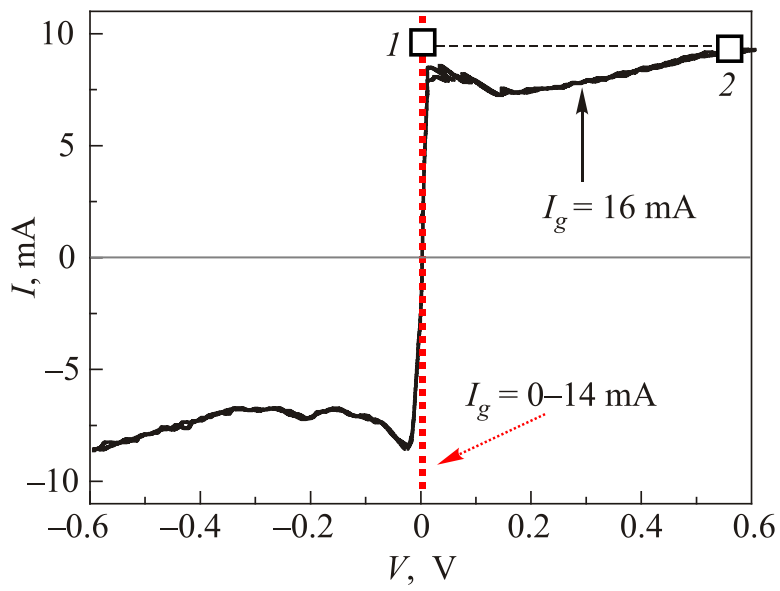

Fig. 4. (Color online) Current-voltage characteristics of a fivelayer MoRe/doped Si/MoRe/doped Si/MoRe heterostructure with a governing current $I_{g}<14 \mathrm{~mA}$ and $I_{g}=16 \mathrm{~mA}$ (dotted and solid lines, respectively). The dashed line demonstrates switching from a zero- (1) to finite-conductance (2) state corresponding to the first resistive state in a MoRe interlayer, see Fig. 2, $T=4.2 \mathrm{~K}$.

perconductor also did not vary remarkably under the external perturbation. The effect was reproduced after thermal cycling above $T_{C}$.

Let us now discuss the five-layer MoRe/doped $\mathrm{Si} / \mathrm{MoRe} /$ doped Si/MoRe devices. We have studied the effect of a dissipation state in a MoRe interlayer on the current-voltage characteristic of the entire structure. Without a governing current $I_{g}$ through the middle interlayer and up to $14 \mathrm{~mA}$, we have observed a superconducting state with very low dissipation (dotted line in Fig. 4). When the $I_{g}$ current was increased up to a value corresponding to the first resistive state in the $I-V$ curve of superconducting MoRe-alloy interlayer (Fig. 2) the character of the current-voltage curve has been changed (Fig. 4). It means that the presence of a non-superconducting plate within the weak link in a multilayered structure with superconducting electrodes strongly influences the charge transport, possibly by creating an extra PS center on the way of Cooper pairs from one electrode to another. Of course, this issue requires further study of the transferring mechanism. From an applied viewpoint, the effect can be used for switching from a zero- to finite-conductance regime driven by transition from a pure superconducting to a first resistive state in a middle MoRe interlayer. Such device can be regarded as a realization of a current-controlled weak-link superconducting contact of the type $b$ proposed and studied in Ref. 19 where a superconducting order parameter in a weak link is changed by managing the current $I_{g}$ in a transverse $\mathrm{S}$ film. The structure discussed above can also serve as a prototype for studying basic properties of layered high-temperature superconductors [20].

\section{Conclusions}

The obtained results evidence in favor of the emergence of phase-slip events in weak links connecting two superconducting plates as was proposed in the recent paper [12]. We have fabricated two types of multilayered structures with two and three $\mathrm{S}$ layers divided by a nanoscale semiconducting (a mixture of Si and Si oxide) film with metallic (W) nanorods between two adjacent superconductors, MoRe-alloy films with the critical temperature of about $9 \mathrm{~K}$. In the case of MoRe/doped Si/MoRe trilayers, we have revealed the emergence of phase-slip events after their irradiation by a high-frequency field. We have followed the arguments of Refs. 11 and 16 where it was argued that the main factor of the high-frequency radiation effect is pair-breaking by its magnetic field. As was shown recently, in the presence of weak and homogeneous disorder, the superconducting state becomes increasingly granular by high magnetic-field application [21]. If so, we can expect that the PS centers may develop in the weak links connecting two $\mathrm{S}$ electrodes at points with weakest superconductivity.

For MoRe/doped Si/MoRe/doped Si/MoRe devices, we have revealed an unexpected effect of a dissipation state in a MoRe interlayer on the $I-V$ characteristics of the entire five-layer structure. It has exhibited itself as switching from a superconducting state with low dissipation to a finite-conductance regime which, in our opinion, can be initiated by emergence of an extra PS center in the interlayer. The latter effect may be caused by a nonequilibrium population of energy levels in the middle film due to multiple Andreev reflections of quasiparticles which induces the appearance of a phase slip region [22,23].

At last, we would like to emphasize that according to Ref. 24 the appearance of phase difference by $2 \pi$ over the nanowire is the dual process to Cooper-pair tunneling in a Josephson junction and hence, by analogy to the Josephson voltage standard, a quantum phase-slip device can realize the fundamental current standard. We hope that the Josephson heterostructures with strongly disordered weak-link interspace proposed in our previous works $[4,7,25]$ and developed in this paper can be used as a prototype of such quantum devices.

This contribution is dedicated to the memory of Professor Ihor Dmytrenko whose pioneering work on the fundamental and applied aspects of weak superconductivity, in particular, Refs. 8 and 13 cited above, have become and still are the basis of our activities in this field.

The work was supported by the Ukrainian-French grant from the MES of Ukraine (Project M/27-2017) and French-Ukrainian Partenariat Hubert Curien (PHC) DNIPRO No. 37984RL. E.Zh. and M.B. are thankful to the support of the Fundamental Research Programme funded by the MES of Ukraine (Project No. 0117U002360) and the grant from the State Fund for Fundamental Research of 
Ukraine (Project F76/65-2017). A.K. is thankful to the support of the Targeted Research \& Development Initiatives Programme funded by the STCU and the National Academy of Sciences of Ukraine (Project No. 6250).

1. A.W. Kleinsasser, in: Heterostructures on Si: One Step Further with Silicon, Y. Nissim and R. Rosencher (eds.), Kluwer (1989).

2. V. Shaternik, A. Shapovalov, M. Belogolovskii, O. Suvorov, S. Döring, S. Schmidt, and P. Seidel, Mater. Res. Exp. 1, 026001 (2014).

3. V.E. Shaternik, A.P. Shapovalov, A.Yu. Suvorov, N.A. Skorik, and M.A. Belogolovskii, Fiz. Nizk. Temp. 42, 544 (2016) [Low Temp. Phys. 42, 426 (2016)].

4. V.E. Shaternik, A.P. Shapovalov, T.A. Prikhna, O.Yu. Suvorov, M.A. Skorik, V.I. Bondarchuk, and V.E. Moshchil, IEEE Trans. Appl. Supercond. 27, 1800507 (2017).

5. D. Olaya, B. Baek, P.D. Dresselhaus, and S.P. Benz, IEEE Trans. Appl. Supercond. 18, 1797 (2008).

6. A.L. Gudkov, M.Yu. Kupriyanov, and A.N. Samus, Zh. Eksp. Teor. Fiz. 141, 939 (2012) [JETP 114, 818 (2012)].

7. V.E. Shaternik, A.P. Shapovalov, and A.Yu. Suvorov, Fiz. Nizk. Temp. 43, 1094 (2017) [Low Temp. Phys. 43, 877 (2017)].

8. I.M. Dmitrenko, Fiz. Nizk. Temp. 22, 849 (1996) [Low Temp. Phys. 22, 648 (1996)].

9. M. Tinkham, Introduction to Superconductivity, 2nd ed., McGraw-Hill Book Co. (1996).

10. N. Giordano, Phys. Rev. Lett. 61, 2137 (1988).

11. R. Tidecks, Current-Induced Nonequilibrium Phenomena in Quasi-One-Dimensional Superconductors, Springer-Verlag (1990).
12. G. Kimmel, A. Glatz, and I.S. Aranson, Phys. Rev. B 95, 014518 (2017).

13. I.M. Dmitrenko, V.G. Volotskaya, L.E. Musienko, and A.G. Sivakov, Physica B 108, 783 (1981).

14. A.G. Sivakov, A.M. Glukhov, A.N. Omelyanchouk, Y. Koval, P. Müller, and A.V. Ustinov, Phys. Rev. Lett. 91, 267001 (2003).

15. G. Berdiyorov, K. Harrabi, F. Oktasendra, K. Gasmi, A.I. Mansour, J.P. Maneval, and F.M. Peeters, Phys. Rev. B 90, 054506 (2014).

16. B. Damaschke and R. Tideks, Z. Phys. B 77, 17 (1989).

17. T.J. Rieger, D.J. Scalapino, and J.E. Mercereau, Phys. Rev. $B$ 6, 1734 (1972).

18. M. Traving, G. Schindler, and M. Engelhardt, J. Appl. Phys. 100, 094325 (2006).

19. I.O. Kulik, A.N. Omelyanchouk, and E.A. Kelman, Fiz. Nizk. Temp. 3, 1107 (1977) [J. Low Temp. Phys. 3, 537 (1977)].

20. V.M. Svistunov, M.A. Belogolovskii, and A.I. Khachaturov, Usp. Fiz. Nauk 163, 61 (1993).

21. R. Ganguly, I. Roy, A. Banerjee, H. Singh, A. Ghosal, and P. Raychaudhuri, Phys. Rev. B 96, 054509 (2017).

22. V.M. Pan, V.P. Gorishnyak, E.M. Rudenko, V.E. Shaternik, M.V. Belous, S.A. Koziychuk, and F.I. Korzhinsky, Cryogenics 23, 258 (1983).

23. V.E. Shaternik, S.Yu. Larkin, and T.A. Khachaturova, Physica C 435, 96 (2006).

24. J.E. Mooij and Y.V. Nazarov, Nature Phys. 2, 169 (2006).

25. V. Lacquaniti, C. Cassiago, N. De Leo, M. Fretto, A. Sosso, P. Febvre, V. Shaternik, A. Shapovalov, O. Suvorov, M. Belogolovskii, and P. Seidel, IEEE Trans. Appl. Supercond. 26, 1100505 (2016). 